\title{
Four cases of echogenic breast lesions: a case series and review
}

Lin Wah $\underline{G o h}^{1}$, MBBS, FRCR, Su Lin Jill Wong ${ }^{2}$, MBChB, FrCR, Puay Hoon $\underline{T a n}^{3}$, MBBS, FRCPA

\begin{abstract}
Echogenic breast lesions are generally thought to be benign. We herein report four cases of echogenic breast lesions that were seen at our centre over 38 months. One patient had a prior history of wide excision and radiotherapy for breast cancer and was imaged as part of routine cancer surveillance, while the other three were recalled for further assessment following an abnormal screening mammogram. All four patients were assessed on ultrasonography, which demonstrated an echogenic lesion in each patient. All four lesions underwent ultrasonography-guided core biopsy, followed by excision biopsy. The indications for biopsy were interval increase in the size of lesion or indeterminate features demonstrated in the lesion. Three lesions were benign, while the lesion from the patient who had received previous radiotherapy was angiosarcoma. Not all echogenic lesions are benign and lesions with suspicious features on ultrasonography should undergo biopsy.
\end{abstract}

Keywords: angiolipoma, angiosarcoma, echogenic breast lesions, myofibroblastoma

\section{INTRODUCTION}

According to the ultrasonography lexicon of the Breast ImagingReporting and Data System of the American College of Radiology, ${ }^{(1)}$ an echogenic breast lesion is defined as a lesion that is hyperechoic in comparison to the surrounding subcutaneous adipose tissue on ultrasonography. Echogenic breast lesions make up a small proportion $(0.6 \%-7 \%)$ of sonographically-detected lesions; ${ }^{(2,3)}$ the overwhelming majority of hyperechoic lesions are benign. Stavros et al reported that hyperechogenicity was the feature with the highest (100.0\%) negative predictive value for malignancy. ${ }^{(2)}$ In a more recent study by Linda et al, out of 1,849 biopsied malignant lesions, only 9 (0.5\%) were hyperechoic. ${ }^{(3)}$ However, patients who have received radiotherapy for breast cancer are known to have an increased risk of radiation-induced malignancies such as invasive ductal carcinoma, lymphoma and angiosarcoma.

Thus far, no data has been published regarding the incidence and histology of echogenic breast lesions in Singapore. We report four cases of echogenic breast lesions that were seen in our institution over a period of 38 months from October 2010 to November 2013. One of the patients had previous wide excision and radiotherapy for breast cancer, while the other three were recalled for further examination following an abnormal screening mammogram.

\section{Case 1}

A 40-year-old Filipino woman had previous right wide excision and axillary clearance in 2006 for invasive ductal carcinoma. She subsequently underwent chemotherapy, hormonal therapy and radiotherapy. She presented with a breast lump near the surgical site in the right upper outer quadrant in November 2013; it was mammographically occult (Figs. 1a \& b). Ultrasonography of the right breast showed a $3.3 \mathrm{~cm}$ hyperechoic mass at the ' 9 o' clock' position, $4.5 \mathrm{~cm}$ from the nipple, with irregular margins (Fig. 1c).
Core biopsy was performed. The histology was atypical vascular proliferation and excision biopsy was recommended. Following excision biopsy, the final histology was well-differentiated angiosarcoma measuring $20 \mathrm{~mm}$ in size (Figs. 1d \& e). The case was discussed at the multidisciplinary meeting and the decision was made to perform right mastectomy with axillary clearance, as the tumour was close to the resection margins. Computed tomography, performed after the mastectomy, did not show any evidence of metastatic disease or lymphadenopathy. At the latest follow-up, the patient was well.

\section{Case 2}

A 51-year-old Chinese woman was recalled for assessment following her third screening episode. A nodule in her left breast, which had been seen during the first screening episode, had increased from $4 \mathrm{~mm}$ to $9 \mathrm{~mm}$ (Figs. 2a \& b). Ultrasonography demonstrated a mixed echo nodule with an echogenic rim (Fig. 2c). Needle biopsy yielded a vascular lesion that was possibly a capillary or lobular haemangioma. Although the tissue obtained was benign, excision was recommended due to the possibility of a more sinister vasoformative lesion in portions that were not sampled. Following excision, a benign angiolipoma was confirmed (Figs. 2d \& e). The patient was subsequently discharged back into the screening programme.

\section{Case 3}

A 73-year-old Chinese woman was recalled following her first screening mammogram for evaluation of right-sided breast nodules (Figs. 3a \& b). Ultrasonography showed a bilobed hyperechoic lesion in the lower inner quadrant of the right breast (Fig. 3c). Needle biopsy revealed a lipovascular lesion. At the multidisciplinary meeting, excision biopsy was recommended for complete histological review in order to exclude more ominous

${ }^{1}$ Department of Diagnostic Radiology, Khoo Teck Puat Hospital, 2Department of Oncologic Imaging, National Cancer Centre Singapore, ${ }^{3}$ Department of Pathology, Singapore General Hospital, Singapore

Correspondence: Dr Goh Lin Wah, Registrar, Department of Diagnostic Radiology, Khoo Teck Puat Hospital, 90 Yishun Central, Singapore 768828. goh.lin.wah@alexandrahealth.com.sg 

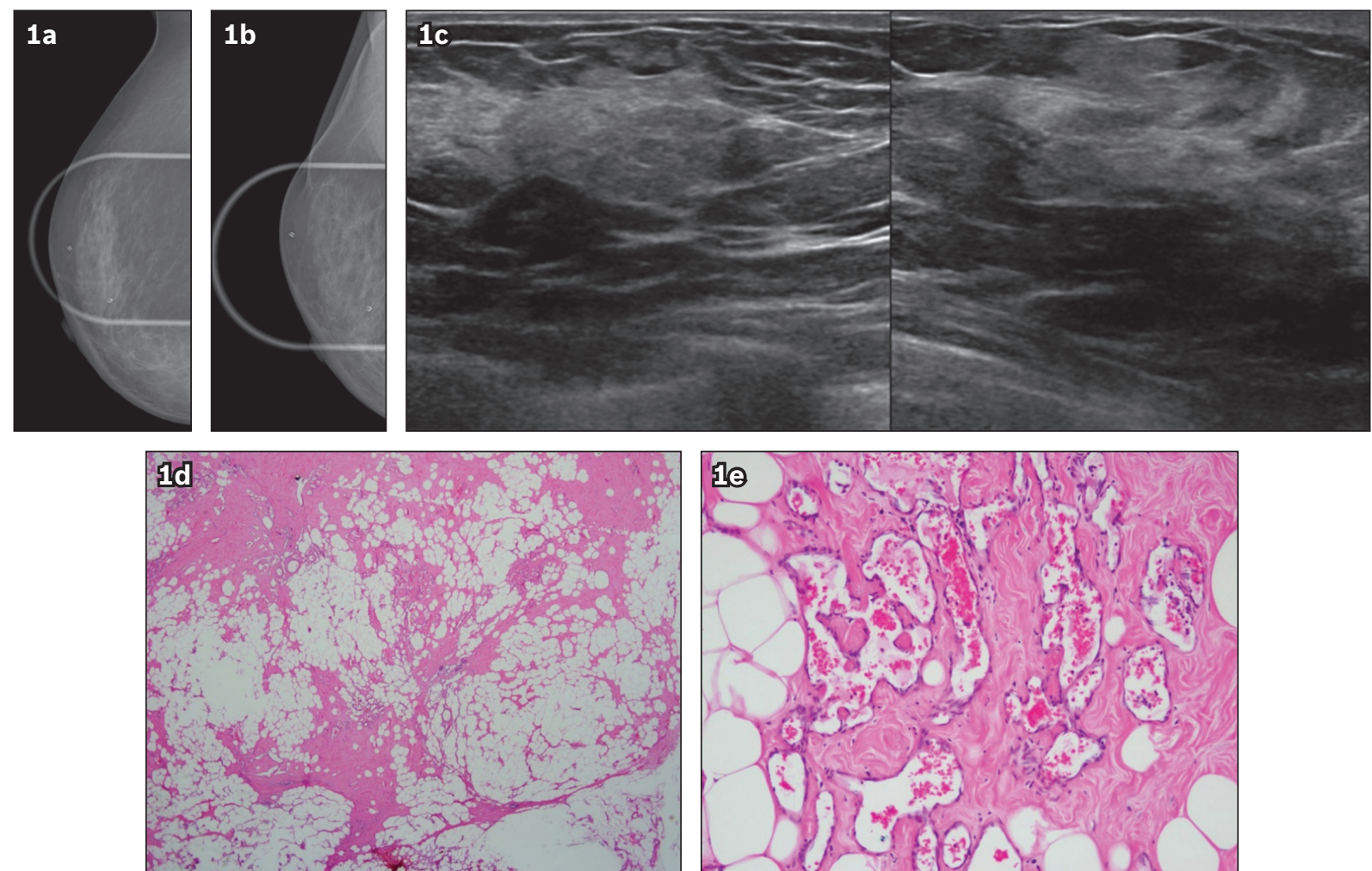

Fig. 1 Case 1: ( $\mathrm{a} \& \mathrm{~b}$ ) Coned compression cranial-caudal and mediolateral-oblique mammogram images of the upper outer quadrant of the right breast obtained after ultrasonography (a marker was placed over the lump seen on the US image) show no demonstrable density or calcification. (c) US images show an irregular echogenic mass at the ' 9 o'clock' position, $4.5 \mathrm{~cm}$ from the nipple. No internal vascularity was seen. Photomicrographs show (d) ectatic vessels containing red blood cells dispersed within the fibroadipose breast parenchyma (Haematoxylin \& eosin, low magnification); and (e) luminal protuberances into the vascular space, which is lined by relatively bland and flattened endothelial cells; the vessels extend into the interstices of the adipose stroma (Haematoxylin \& eosin, high magnification).
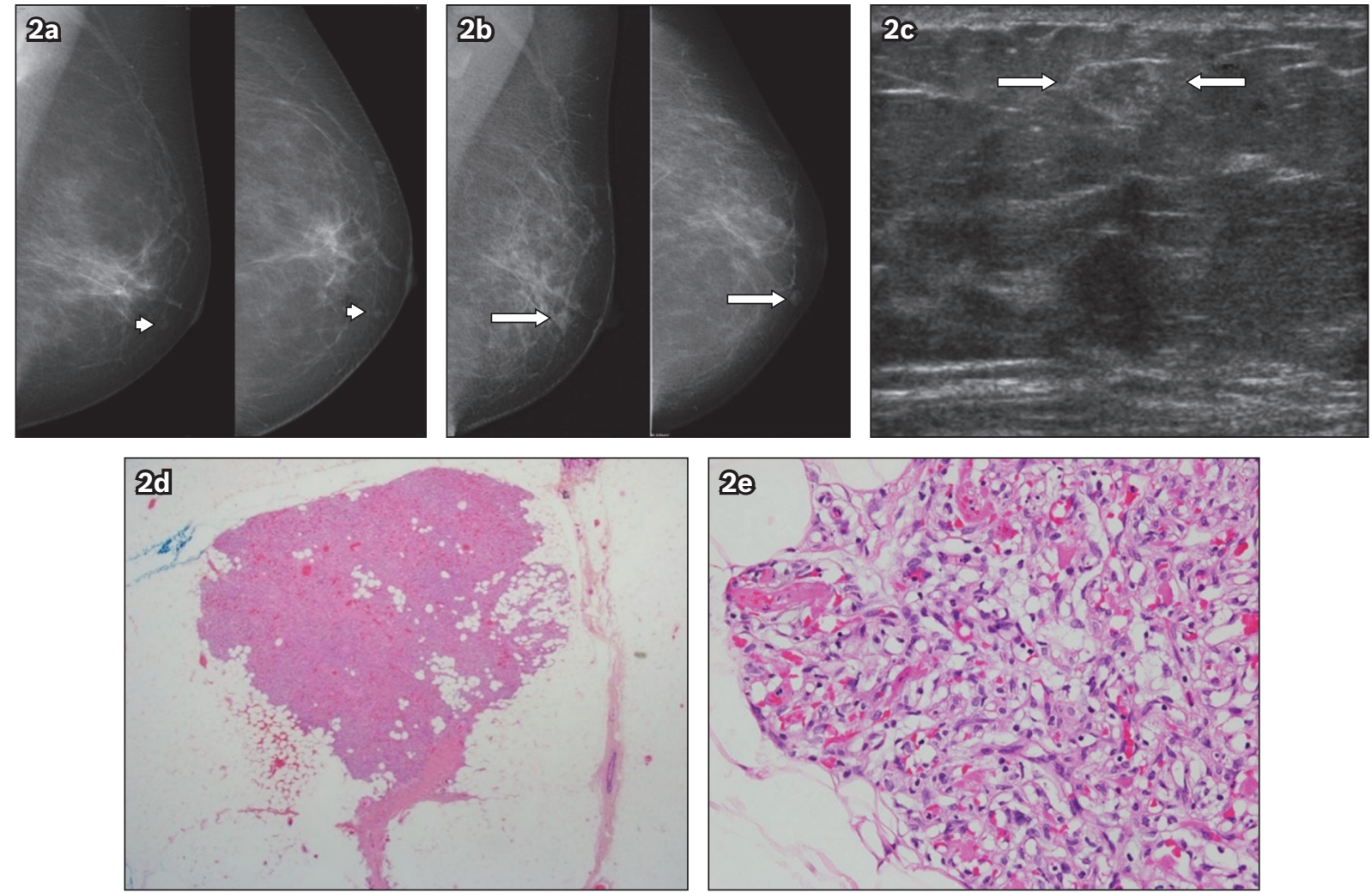

Fig. 2 Case 2: (a) Mediolateral-oblique (MLO) and cranial-caudal (CC) screening mammogram images obtained in 2008 show a tiny nodule (arrowheads) in the lower medial quadrant. (b) MLO and CC screening mammogram images obtained in 2010 show a well-defined nodule (arrows) in the lower medial quadrant; the nodule, when compared to the one seen in 2008, has increased in size. (c) US image reveals a heterogeneous nodule with an echogenic rim (between arrows). Photomicrographs show (d) a tight collection of congested capillaries, with a partially circumscribed boundary (Haematoxylin \& eosin, low magnification); and (e) several fibrin thrombi within the capillaries of the angiolipoma (Haematoxylin \& eosin, high magnification). 

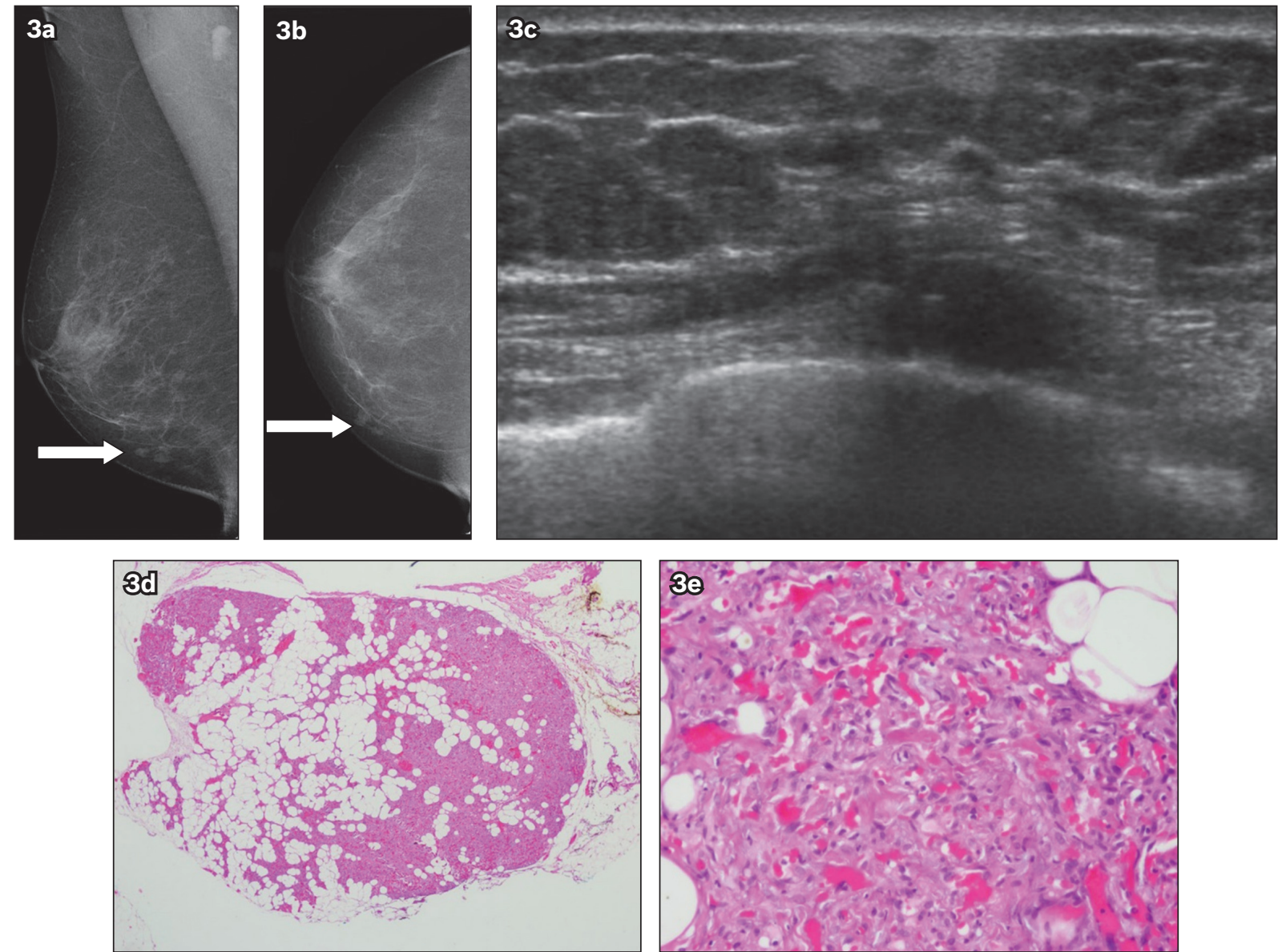

Fig. 3 Case 3: (a \& b) Mediolateral-oblique and cranial-caudal screening mammogram images show nodular densities (arrows) in the lower medial aspect of the right breast. (c) US image shows a predominantly hyperechoic mass in the lower inner quadrant. Photomicrographs show (d) a circumscribed lesion composed of an admixture of mature adipocyte lobules, with tight congeries of congested capillaries (Haematoxylin \& eosin, low magnification); and (e) a few fibrin thrombi within the capillaries (Haematoxylin \& eosin, high magnification).

pathology, such as angiosarcoma. Surgical excision confirmed a benign angiolipoma (Figs. 3d \& e). The patient was discharged back into the routine screening programme.

\section{Case 4}

A 46-year-old Malay woman was recalled for reassessment of an enlarging mass in the left breast. Two years earlier, she was assessed for the same mass, which measured $30 \mathrm{~mm} \times 13 \mathrm{~mm}$. As it was felt to be benign on clinical examination and imaging, she was discharged without biopsy. In 2010, the patient was recalled for repeat assessment, as the mass had increased in size (Figs. 4a \& b). Ultrasonography demonstrated an echogenic lump in the left ' $12 \mathrm{o}^{\prime}$ ' lock' position (Fig. 4c), similar to that seen in the earlier assessment. However, there was interval increase in size to $40 \mathrm{~mm} \times 19 \mathrm{~mm}$. Ultrasonography-guided needle biopsy was performed, demonstrating a benign fibrous tissue with features indicating possible myofibroblastoma. Subsequently, excision biopsy was performed in view of a significant increase in the size of the mass, which confirmed this diagnosis (Figs. $4 \mathrm{~d} \& \mathrm{e}$ ). The patient was discharged back to the screening programme.

\section{DISCUSSION}

A differential diagnosis for echogenic breast lesions, ${ }^{(4)}$ both benign and malignant, can be encountered in daily practice
Table I. Differential diagnosis of an echogenic breast mass.

\begin{tabular}{ll}
\hline Type of lesion & Differential diagnosis \\
\hline Benign & - Haematoma \\
& - Fat necrosis \\
& - Haemangioma \\
& - Angiolipoma \\
& - Hamartoma \\
& - Myofibroblastoma \\
- Invasive ductal carcinoma & - Ductal carcinoma in situ \\
Malignant & - Invasive lobular carcinoma \\
& - Angiosarcoma \\
\hline
\end{tabular}

(Table I). Stavros et al ${ }^{(2)}$ reported that hyperechogenicity was the feature with the highest $(100.0 \%)$ negative predictive value for malignancy. In a more recent study by Linda et al, only $9(0.5 \%)$ out of 1,849 biopsied malignant lesions were echogenic; the study also found that lesion orientation and margins proved to be significantly different between malignant and benign nodules. ${ }^{(3)}$ Malignant lesions were more likely to have uncircumscribed margins and nonparallel orientations compared to benign lesions. The ultrasonography findings have to be carefully interpreted in conjunction with the mammographic findings 

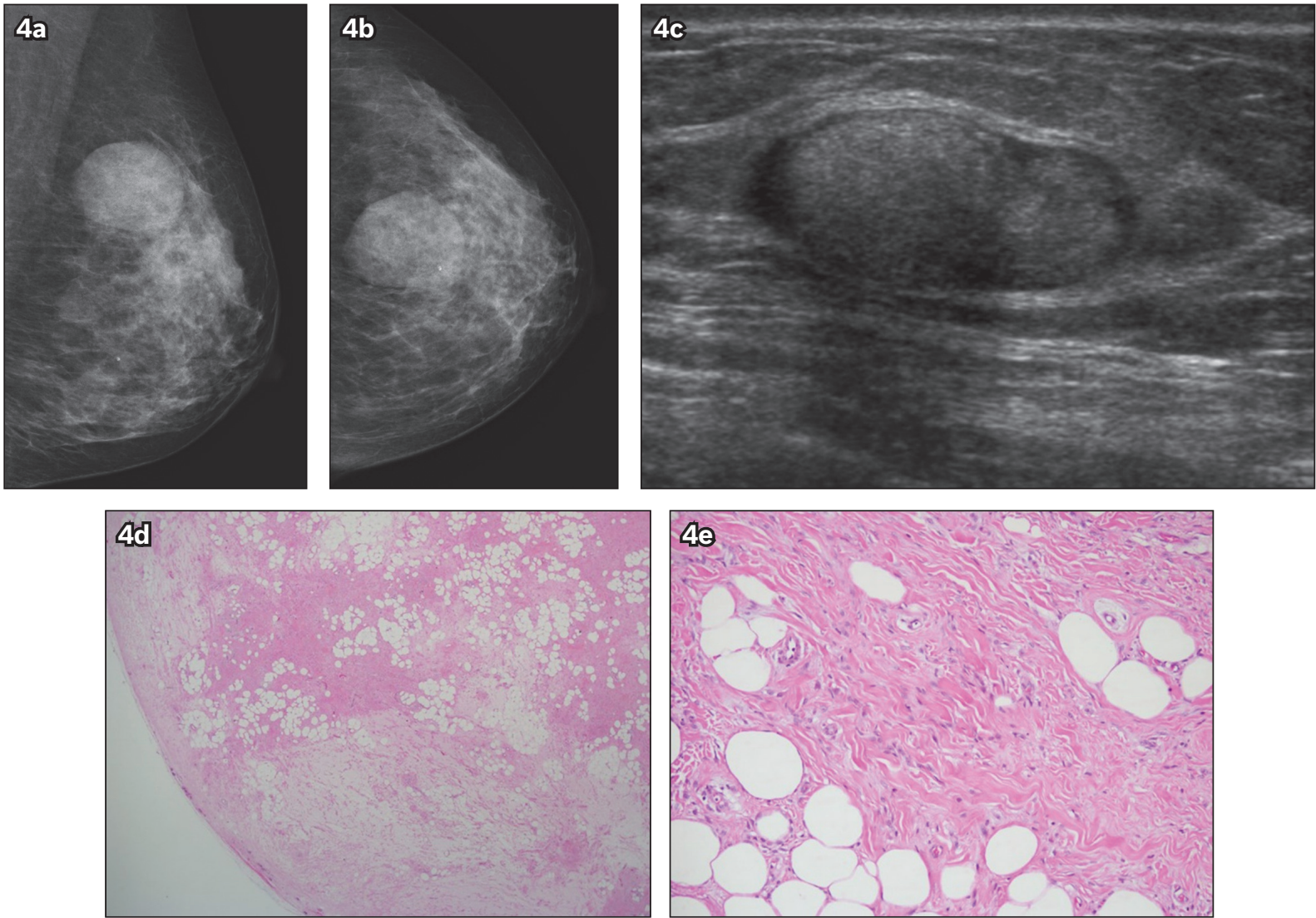

Fig. 4 Case 4: (a \& b) Mediolateral-oblique and cranial-caudal screening mammogram images show a well-defined mass in the upper outer quadrant of the left breast; the mass was larger than the mass observed in the mammogram images obtained two years earlier. (c) US image shows a well-defined mass with an echogenic centre and a hypoechoic rim. Photomicrographs show (d) the border of the myofibroblastoma, with a few small lobules near the periphery; the lobules of mature adipocytes are interspersed with fibrous zones that are variably oedematous (Haematoxylin \& eosin, low magnification); and (e) bland spindle cell nuclei among pink collagen bands (Haematoxylin \& eosin, high magnification).

and an understanding of the patient's clinical background. Clinical history of trauma along with mammographic findings of a dense mass may still represent haematoma or fat necrosis, which can be followed up. An echogenic mass that is lucent on mammography is most likely benign. However, a mass that demonstrates increased asymmetry, spiculated margins, interval enlargement, associated microcalcifications or lymphadenopathy on mammography typically requires biopsy regardless of the patient's history. ${ }^{(5)}$

In Case 1, the patient had a medical history of breast carcinoma and irradiation to the breast; hence, a presenting complaint of a new palpable lump in this scenario would be highly suspicious for a malignant lesion. Possible considerations include local recurrence or a lesion secondary to previous treatment (e.g. fat necrosis). Secondary angiosarcomas are known to occur most frequently after breast conservation therapy, with an average latency of 5-6 years. ${ }^{(6)}$ The incidence of post-irradiation angiosarcoma is low, ranging from $0.09 \%$ to more than $0.30 \% .{ }^{(7-9)}$ Our patient had an interval of eight years since her previous breast surgery and treatment. Mammographically and sonographically, the skin-thickening from the dermal lesions can be masked by postradiation skin thickening. Intraparenchymal masses can present as heterogeneous lesions with alteration of normal soft tissue planes. ${ }^{(10)}$ Secondary angiosarcomas tend to have poor prognosis and the outcome is dependent on complete surgical resection of the lesion. Hence, these lesions need to be identified early while they are resectable. Although the lesion in our patient did not demonstrate abnormal vascularity on colour flow imaging, its margins were irregular and it was assessed as an indeterminate lesion requiring biopsy.

Cases 2-4 did not have any previous history of malignancy. However, the temporal evolution of the lesion in Case 2 was worrisome, as on second screening mammogram, the nodule had increased in size. The mammographic findings in Case 3 were dense nodules, which on ultrasonography showed indistinct margins, warranting biopsy. Both Cases 2 and 3 yielded vascular tissue on needle biopsy and, although rare, angiosarcoma (one of the malignancies that demonstrates echogenicity) needed to be ruled out; both patients' lesions were confirmed to be angiolipomas on excision biopsy. Angiolipomas are unusual fat-containing tumours with mature adipocytes interposed with variable vascular proliferation. ${ }^{(11)}$ They usually present in the trunk and extremities, and less often in the breast. Our review of the literature found several previous case reports on angiolipomas of the breast, ${ }^{(11-17)}$ with one paper describing up to 52 cases. ${ }^{(18)}$ The histologic hallmark of this lesion is scattered microthrombi in small blood vessels. ${ }^{(19)}$ Angiolipomas do not have characteristic imaging findings and frequently overlap with benign and malignant 
lesions. ${ }^{(20)}$ They have been described as oval or round isodense masses on mammography ${ }^{(4,21)}$ due to the fatty components that have a variable angiomatous component. Sonographically, angiolipomas can present as round or oval masses in the subcutaneous tissue that are isoechoic or hyperechoic, but they are rarely hypoechoic with an echogenic rim. Due to the nonspecific radiological features, biopsy is usually required for diagnosis. ${ }^{(4)}$

Case 4 was another screen-detected lesion and, similar to Case 2, this lesion showed interval increase in size over two years. Although the patient's mammographic and sonographic findings were relatively benign in appearance, with a well-defined and encapsulated echogenic lesion, the decision to perform biopsy was made in view of the interval growth. The final histology of the lesion was that of a benign myofibroblastoma, an unusual tumour that most often affects elderly men and postmenopausal women. ${ }^{(4,22)}$ Not many cases of myofibroblastomas have been reported in the literature; one other reported case occurred in an elderly male patient. ${ }^{(22)}$ This tumour is histologically well-demarcated and composed of bipolar spindle cells among structured collagen bundles. ${ }^{(22)}$ The radiologic features of myofibroblastomas are variable. ${ }^{(23)}$ They are usually oval, well-defined, benign-looking on mammograms and rarely associated with calcifications. ${ }^{(23)}$ Sonography findings may overlap with that of a fibroadenoma, as they both have circumscribed margins and variable echogenicity. Core biopsy is usually required for diagnosis and treatment is by local excision. ${ }^{(4)}$

The four cases above illustrate the difficulties in managing echogenic breast lesions in daily clinical practice. Their sonographic features are not entirely benign. Two cases had poorly circumscribed borders, whereas the two other cases demonstrated a progressive increase in size. Thus, the presence of significant hyperechoic elements in a lesion is suggestive but not conclusive for benignity. Excision biopsy may be warranted, despite benign needle biopsy results, especially if prompted by careful multidisciplinary review.

\section{REFERENCES}

1. American College of Radiology. ACR BI-RADS: ultrasound. In: ACR Breast Imaging Reporting and Data System, Breast Imaging Atlas. Reston, VA: American College of Radiology, 2003.

2. Stavros AT, Thickman D, Rapp CL, et al. Solid breast nodules: use of sonography to distinguish between benign and malignant lesions. Radiology 1995; 196:123-34.

3. Linda A, Zuiani $C$, Lorenzon $M$, et al. Hyperechoic lesions of the breast: not always benign. AJR Am J Roentgenol 2011; 196:1219-24.

4. Adrada B, Wu Y, Yang W. Hyperechoic lesions of the breast: radiologichistopathologic correlation. AJR Am J Roentgenol 2013; 200:W518-30.

5. Harvey JA, Nicholson BT, Cohen MA. Finding early invasive breast cancers: a practical approach. Radiology 2008; 248:61-76.

6. Brenn T, Fletcher CD. Postradiation vascular proliferations: an increasing problem. Histopathology 2006; 48:106-14.

7. Marchal C, Weber B, de Lafontan B, et al. Nine breast angiosarcomas after conservative treatment for breast carcinoma: a survey from French comprehensive Cancer Centers. Int J Radiat Oncol Biol Phys 1999; 44:113-9.

8. Strobbe LJ, Peterse HL, van Tinteren H, Wijnmaalen A, Rutgers EJ. Angiosarcoma of the breast after conservation therapy for invasive cancer, the incidence and outcome. An unforeseen sequel. Breast Cancer Res Treat 1998; 47:101-9.

9. West JG, Qureshi A, West JE, et al. Risk of angiosarcoma following breast conservation: a clinical alert. Breast J 2005; 11:115-23.

10. Chen KT, Kirkegaard D, Bocian JJ. Angiosarcoma of the breast. Cancer 1980; 46:368-71.

11. Weinstein SP, Conant EF, Acs G. Case 59: Angiolipoma of the breast. Radiology 2003; 227:773-5.

12. Cheung $\mathrm{YC}$, Wan $\mathrm{YL}, \mathrm{Ng} \mathrm{SH}$, et al. Angiolipoma of the breast with microcalcification. Mammographic, sonographic, and histologic appearances. Clin Imaging 1999; 23:353-5.

13. Mintz AD, Mengoni P. Angiolipoma of the breast: sonographic appearance of two cases. J Ultrasound Med 1998; 17:67-9.

14. Noel JC, Van Geertruyden J, Engohan-Aloghe C. Angiolipoma of the breast in a male: a case report and review of the literature. Int J Surg Pathol 2011; 19:813-6.

15. Sibala JL, Chang CH, Lin F, Thomas JH. CT of angiolipoma of the breast. AJR Am J Roentgenol 1980; 134:840-1.

16. Yilmaz E, Lebe B, Tuna B, Ozen E. Cellular angiolipoma of the breast with unusual radiologic presentation as a carcinoma. Eur J Radiol Extra 2004; 49:57-9.

17. Kaur N, Gulati A, Kaushik R, Kaushal V. Angiolipoma of the mammary region: A report of two cases. J Health Spec 2013; 1:100-2.

18. Kryvenko ON, Chitale DA, VanEgmond EM, et al. Angiolipoma of the female breast: clinicomorphological correlation of 52 cases. Int J SurgPathol 2011; 19:35-43.

19. Tavassaoli FA. Mesenchymal lesions. In: Pathology of the breast. Norwalk, CT: Appleton \& Lange, 1992:517-60.

20. Gao Y, Slanetz PJ, Eisenberg RL. Echogenic breast masses at US: to biopsy or not to biopsy? Radiographics 2013; 33:419-34.

21. Darling ML, Babagbemi Tom, Smith DN, et al. Mammographic and Sonographic Features of Angiolipoma of the Breast. Breast J 2000; 6:166-170.

22. Mele M, Jensen V, Wronecki A, Lelkaitis G. Myofibroblastoma of the breast: Case report and literature review. Int J Surg Case Rep 2011; 2:93-6.

23. Lee YS, Gilcrease $M, W u Y, Y a n g ~ W T$. Myofibroblastoma of the breast: Imaging features. Eur J Radiol Extra 2010; 73:e13-e15. 